\title{
Quasiclassical dipole matrix elements for high atomic states and stochastic dynamics of hydrogen atoms in microwave fields
}

\author{
B Kaulakys $\dagger$ \\ Institute of Theoretical Physics and Astronomy, Lithuanian Academy of Sciences, \\ K. Poželos 54, 232600 Vilnius, Lithuania, USSR
}

Received 17 May 1990, in final form 10 October 1990

\begin{abstract}
Analytical expressions for dipole matrix elements between high atomic states of non-hydrogenic and perturbed hydrogenic atoms are derived. The theoretical investigation is fulfilled for discrete and continum atomic states and for the one-dimensional atom. It is shown that the quantal transition probabilities between perturbed atomic states depend on the nature of perturbation, e.g. matrix elements for the hydrogenic atom in an electromagnetic field differ from those of the non-hydrogenic atom. The results obtained generalize and increase the accuracy of some known expressions for the non-hydrogenic atom and extend them for the perturbed hydrogenic atom. Further, the analysis of classical dynamics and the derivation of mapping equations of motion for the one-dimensional atom in a harmonic field is fulfilled. The relationship between the energy change of the classical atom in a harmonic field and expressions for dipole matrix elements is revealed and analysed.
\end{abstract}

\section{Introduction}

The progress in experimental laser spectroscopy of high atomic Rydberg states and the great attention being paid to the non-linear and multiphoton ionization requires computation of transition probabilities between non-hydrogenic atomic states of high effective principal quantum numbers $\nu, \nu^{\prime}$. For a hydrogen atom exact quantum mechanical expressions of the dipole matrix elements between non-perturbed states of integer principal quantum numbers $n, n^{\prime}$ are available (Gordon 1929, Bethe and Salpeter 1957). However, their complex analytic structure means that one needs to look for approximations as the quantum numbers grow larger. The semiclassical approximation for the discrete spectrum of hydrogen-like atoms has been performed by Bureeva (1968). The generalization of the semiclassical approximation for electric multipole transitions and the test of the applicability of the JWKB approximation and classical perturbation theory for hydrogenic atoms has been fulfilled by Heim et al (1989).

The extension of the semiclassical formulae for dipole matrix elements to nonhydrogenic Rydberg states using the quantum defect method has been undertaken by Davydkin and Zon (1981) for discrete spectrum and by Delone et al (1989) for continuum states. Although the results of Davydkin and Zon (1981) are widely used they contain some imperfections. The main formulae of this paper contain some terms

$\dagger$ Alexander von Humboldt Foundation Research Fellow 1990/91, I. Physikalisches Institut der Justus-LiebigUniversität, Heinrich-Buff-Ring 16, D-6300 Giessen, Federal Republic of Germany. 
with erroneous sign and thus they do not fulfil some well known exact results, e.g. the limiting result when $s=\nu^{\prime}-\nu \rightarrow 0$.

On the other hand, the considerable interest in the non-linear ionization of the hydrogenic atoms by a microwave field (for review see Casati et al 1987, 1988) requires computation of transition probabilities between states of high fractional effective principal quantum numbers of the perturbed hydrogenic atom. The transitions between strongly perturbed states may be considered either as transitions in the continuum (Gontis and Kaulakys 1987) or as transitions between photonic states of a Rydberg atom in a harmonic field (Casati et al 1987, 1988, Gontis and Kaulakys 1991). These transition amplitudes are related to some characteristics of stochastic dynamics of the hydrogenic atoms in a microwave field such as the diffusion coefficient of the electron in the energy space and the localization length of quantal suppressed diffusion. The diffusion coefficient can be obtained from the mapping form of the classical equations of motion. As was noticed by Gontis and Kaulakys (1987) the energy change of the electron during the period of classical intrinsic motion in the Coulomb field, due to the interaction with a harmonic field and diffusion coefficient expression, depends on the starting condition, i.e. on the integration interval. The local diffusion coefficient in the energy space may be derived on the basis of quantum mechanics as well. It follows from the correspondence principle that the classical and quantum descriptions have as a consequence the same conclusions, i,e. a great number of uncorrelated one-photon transitions result in the diffusion of the electron in the energy space identical to the diffusion due to the stochastic classical motion (Gontis and Kaulakys 1987) $\dagger$. Thus, the analysis of the quantal transition probabilities may clarify some vagueness in the mapping description of the classical dynamics of the hydrogen atom in a microwave field.

In this paper it is shown that the quantal transition probabilities between perturbed hydrogenic atom states with fractional effective principal quantum numbers depend on the nature of perturbation. Thus, for the non-hydrogenic atoms the perturbation of the Coulomb potential is considerable only at small distances while for the hydrogenic atoms in an electromagnetic field the perturbing potential is long range. As a result of this difference the expressions for the dipole matrix elements between atomic states with high effective principal quantum number of the perturbed hydrogenic atom (see equations (23) and (24) below) differ from those for the non-hydrogenic atom (equation (16) below). In addition, the relationship between the dipole matrix elements and the energy change of the classical atom in a harmonic field is revealed and analysed and the mapping equations of motion are derived.

Note that by the term 'non-hydrogenic atom' we understand to mean the real atom, quantum states of which differ from the hydrogenic due to the deviation of the atomic core potential from the Coulomb potential; while the term 'perturbed hydrogenic atom' means the hydrogenic atom in an external field, quantum states of which differ from the hydrogenic due to the perturbation by the external field. Of course, one can examine the quantum transitions between perturbed states of the non-hydrogenic, e.g. alkalimetal atom states. However, the behaviour of the non-hydrogenic atoms in a strong electromagnetic field, e.g. the mechanism of multiphoton ionization, differs from that of the hydrogenic atom (see, e.g. Stoneman et al 1988 and references therein) and, therefore, those problems are beyond the scope of this paper.

$\dagger$ Here we do not discuss the quantum localization phenomenon which is related with correlations of one-photon transitions and manifests four times larger some diffusion time (see Casati et al 1987, 1988). 
The paper is organized as follows. After an introduction we present the analytical expressions for the quasiclassical radial wavefunctions of the non-hydrogenic and perturbed hydrogenic atoms (section 2). In section 3 the derivation and analysis of the quasiclassical dipole matrix elements between high discrete states of the nonhydrogenic and perturbed hydrogenic atoms are given, while sections 4 and 5 are devoted to the dipole matrix elements for the continuum states and one-dimensional atom, respectively. The results of these sections generalize and increase the accuracy of some known expressions for the non-hydrogenic atom and extend them for the perturbed hydrogenic atom. Section 6 is devoted to the analysis of the classical dynamics of the one-dimensional hydrogen atom in a harmonic field, to the connection of the energy change of the classical atom in a harmonic field with expressions for the dipole matrix elements and to the derivation of the iterative (mapping) equations of motion for the classical hydrogen atom in an oscillating electric field. In section 7 some conclusions are drawn.

\section{Quasiclassical approximations for wavefunctions}

Calculation of the matrix elements for the dipole momentum between atomic states requires the radial wavefunctions of the excited atomic states. For high states one can use the quasiclassical approximation. The quasiclassical solution $R_{\nu l}$ of the radial Schrödinger equation for a highly excited atom can be expressed as

$$
P_{v l}=r R_{\nu l}=\left(\frac{2}{\pi \nu^{3} k(r)}\right)^{1 / 2} \cos \left[\int_{r_{1}}^{r} k(r) \mathrm{d} r-\frac{1}{4} \pi\right]
$$

in the classically allowed region of motion and some exponentially decreasing functions outside this region (see e.g. Landau and Lifshitz 1960). The local wavenumber $k(r)$ in equation (1) is given by

$$
k(r)=\left[2 E-2 U(r)-\frac{\left(l+\frac{1}{2}\right)^{2}}{r^{2}}\right]^{1 / 2}
$$

where the potential $U(r)$ which defines the motion of the Rydberg electron may be expressed as a sum of two terms--the Coulomb potential and the perturbation potential $\Delta U(r)$, i.e.

$$
U(r)=-\frac{1}{r}+\Delta U(r)
$$

The energy of state $E$ is, in the usual way, related to the effective principal quantum number $\nu$ and the quantum defect $\mu_{t}$

$$
E=-\frac{1}{2 \nu^{2}} \quad \nu=n-\mu_{1} \text {. }
$$

The roots of the wavenumber $k(r), r_{1}$ and $r_{2}$, are two turning points which delimit the region of the classical motion.

If the perturbation potential $\Delta U(r)$ is small $(|\Delta U| \ll 1 / r)$ the wavenumber $k(r)$ according to equation (2) can be expressed in the form (see Beigman and Bureeva (1981) for an analogy)

$$
k(r)=k_{\mathrm{c}}(r)-\frac{\Delta U(r)}{k_{\mathrm{c}}(r)}
$$


where the local wavenumber for the Coulomb potential

$$
k_{\mathrm{c}}(r)=\frac{\nu}{r}\left[-\left(\frac{r}{\nu}\right)^{2}+\frac{2 r}{\nu^{2}}-\left(\frac{l+\frac{1}{2}}{\nu}\right)^{2}\right]^{1 / 2}=\frac{\varepsilon \sin \xi}{\nu(1-\varepsilon \cos \xi)}
$$

is introduced. For the Coulomb potential it is convenient to introduce the parametric equations of motion

$$
\begin{array}{ll}
r=\nu^{2}(1-\varepsilon \cos \xi) & \varepsilon=\left[1-\left(\frac{l+\frac{1}{2}}{\nu}\right)^{2}\right]^{1 / 2} \\
t=\nu^{3}(\xi-\varepsilon \sin \xi) & r_{1,2}^{\mathrm{c}}=\nu^{2}(1 \mp \varepsilon)
\end{array}
$$

here $\varepsilon$ denotes the eccentricity of the classical orbit and $\xi(-\infty<\xi<\infty)$ is the parameter.

The quasiclassical quantization condition for the excited state can be expressed as

$$
\int_{r_{1}}^{r_{2}} k(r) \mathrm{d} r=\left(n_{\mathrm{r}}+\frac{1}{2}\right) \pi
$$

where the radial quantum number, $n_{\mathrm{r}}=n-l-1$, is equal to the number of zeros of the radial wavefunction. According to equations (3)-(7) we have

$$
\int_{r_{1}^{c}}^{r_{2}^{c}} k_{c}(r) d r=\left(\nu-l-\frac{1}{2}\right) \pi \quad-\int_{r_{1}}^{r_{2}} \frac{\Delta U(r) \mathrm{d} r}{k_{\mathrm{c}}(r)}=\delta_{l}
$$

with $\delta_{l}$ being the non-Coulomb scattering phase related to the quantum defect $\mu_{l}$ by the relationship $\delta_{l}=\pi \mu_{l}$.

Finally, the radial part of the perturbed hydrogenic or non-hydrogenic atom wavefunction may be approximated as

$$
P_{\nu l}=\left(\frac{2}{\pi \nu^{3} k_{\mathrm{c}}(r)}\right)^{1 / 2} \cos \left[\int_{\mathrm{r}_{\mathrm{i}}}^{r} k_{\mathrm{c}}(r) \mathrm{d} r+\delta_{l}(r)-\frac{1}{4} \pi\right]
$$

where

$$
\delta_{l}(r)=-\int_{r_{l}}^{r} \frac{\Delta U(r)}{k_{\mathrm{c}}(r)} \mathrm{d} r \quad \delta_{l}=\delta_{l}\left(r_{2}\right) .
$$

Note that using equations (7) and (8), the initial equation (1) for the radial wavefunction can be written as

$$
P_{\nu l}(r)=(-1)^{n-1-1}\left(\frac{2}{\pi \nu^{3} k(r)}\right)^{1 / 2} \cos \left[\int_{r_{2}}^{r} k(r) \mathrm{d} r+\frac{1}{4} \pi\right]
$$

which contains the right sign of the asymptotic at $r \rightarrow r_{2}$ (see, e.g. Landau and Lifshitz $1960) \dagger$.

\section{Quasiclassical dipole matrix elements for discrete states}

The radial dipole matrix element of a transition $\nu l \rightarrow \nu^{\prime} l^{\prime}$ is given by the well known expression

$$
\left\langle\nu l|r| \nu^{\prime} l^{\prime}\right\rangle=\int_{0}^{\infty} P_{\nu l}(r) P_{\nu^{\prime} l^{\prime}}(r) r \mathrm{~d} r
$$

$\dagger$ One should note the missing phase in the analogous equation (2) of the paper by Davydkin and Zon (1981) which results in the erroneous signs of the main final expressions in that paper. 
Our calculations of the matrix element (12) are similar to those by Heim et al (1989). Substituting equation (9) into equation (12) one replaces the product of the two cosines by half the sum of the cosines of the difference and the sum of their arguments. Neglecting the rapidly oscillating cosine of the sum of the phases and holding only linear terms in the expansion of the difference of the phases in powers of $s=\nu^{\prime}-\nu$ and $\Delta=l^{\prime}-l$ one finally finds that

$$
\begin{aligned}
\left\langle\nu l|r| \nu^{\prime} l^{\prime}\right\rangle=\frac{\nu \nu^{\prime}}{\pi} & \int_{0}^{\pi}(1-\varepsilon \cos \xi)^{2} \\
& \times \cos \left[s(\xi-\varepsilon \sin \xi)-\Delta l \cos ^{-1}\left(\frac{\cos \xi-\varepsilon}{1-\varepsilon \cos \xi}\right)+\Delta(\xi)\right] \mathrm{d} \xi
\end{aligned}
$$

where

$$
\begin{aligned}
& \Delta(\xi)=\delta_{l}(\xi)-\delta_{l}(\xi) \quad \xi=\cos ^{-1}\left(\frac{\nu^{2}-r}{\varepsilon \nu^{2}}\right) \\
& \varepsilon=\left[1-\frac{\left(l+l^{\prime}+1\right)^{2}}{4 \nu \nu^{\prime}}\right]^{1 / 2} .
\end{aligned}
$$

For the dipole transitions, $\Delta l= \pm 1$ and that is why equation (13) reduces to

$$
\begin{aligned}
\left\langle\nu l|r| \nu^{\prime} l \pm 1\right\rangle= & \frac{\nu \nu^{\prime}}{\pi} \int_{0}^{\pi}\{\cos [s(\xi-\varepsilon \sin \xi)+\Delta(\xi)](\cos \xi-\varepsilon) \\
& \left. \pm \sqrt{1-\varepsilon^{2}} \sin [s(\xi-\varepsilon \sin \xi)+\Delta(\xi)] \sin \xi\right\}(1-\varepsilon \cos \xi) \mathrm{d} \xi
\end{aligned}
$$

Later in this section we will analyse the different special cases of equation (15), i.e. we will derive expressions for the matrix elements of the non-hydrogenic atom and of the hydrogenic atom in an external field.

\subsection{Non-hydrogenic Rydberg atoms}

For the non-hydrogenic atoms the potential which defines the motion of the Rydberg electron deviates appreciably from the Coulomb potential only at small distances, i.e. the perturbation potential $\Delta U(r)$ is short-range, while the main contribution to the radial integral (15) occurs at large distances, i.e. $r \sim \nu^{2}$. Thus, in equation (15) the difference of the non-Coulomb scattering phases $\Delta(\xi)$ may be replaced by the constant $\Delta=\delta_{l}-\delta_{l}$. Therefore, integration of equation (15) by parts yields

$$
\begin{aligned}
& \left\langle\nu l|r| \nu^{\prime} l \pm 1\right\rangle=(-1)^{\Delta n} \frac{\nu \nu^{\prime}}{s}\left[\mathrm{~J}_{-s}^{\prime}(s \varepsilon)+\frac{1-\varepsilon}{\pi} \sin s \pi \pm \sqrt{\varepsilon^{-2}-1}\left(\mathrm{~J}_{-s}(s \varepsilon)-\frac{\sin s \pi}{s \pi}\right)\right] \\
& \Delta n=n^{\prime}-n \quad s=\nu^{\prime}-\nu .
\end{aligned}
$$

Here $J_{s}(z)$ and $J_{s}^{\prime}(z)$ are the Anger function and its derivative with respect to the argument 2 . In addition, the identity $\Delta=\pi(\Delta n-s)$ was used. Equation (16) with accuracy to the signs of some terms coincides with equation (9) by Davydkin and Zon (1981). Let us analyse equation (16) more carefully.

The expansion of the functions $\mathrm{J}_{ \pm s}(s \varepsilon)$ and $\mathrm{J}_{ \pm s}^{\prime}(s \varepsilon)$ in powers of $s$ are (Watson 1958)

$$
\begin{array}{ll}
\mathrm{J}_{\mp s}(s \varepsilon)=\frac{\sin s \pi}{s \pi\left(1-s^{2}\right)}\left[1-s^{2}\left(1 \pm \frac{1}{2} \varepsilon\right)^{2}\right] & s \leqslant 1 \\
\mathrm{~J}_{\mp s}^{\prime}(s \varepsilon)=\frac{\sin s \pi}{\pi\left(1-s^{2}\right)}\left[\mp 1-\frac{1}{2} \varepsilon+\frac{1}{48} s^{2} \varepsilon\left(18 \pm 16 \varepsilon+3 \varepsilon^{2}\right)\right] & s \leqslant 1 .
\end{array}
$$


Thus, the limiting case of equation (16) for $n^{\prime}=n(s=0)$ results in the exact matrix element of the hydrogenic atom (see e.g. Heim et al 1989) $\dagger$

$$
\langle n l|r| n l \pm 1\rangle=-\frac{3}{2} n^{2} \varepsilon .
$$

For the integers $s=\Delta n \neq 0,(-1)^{\Delta n} \mathrm{~J}_{-\Delta n}(\Delta n \varepsilon)=J_{\Delta n}(\Delta n \varepsilon)$ and $(-1)^{\Delta n} \mathrm{~J}_{-\Delta n}^{\prime}(\Delta n \varepsilon)=$ $\mathrm{J}_{\Delta n}^{\prime}(\Delta n \varepsilon)$ where $J_{i},(z)$ is the Bessel function. So equation (16) reduces to the quasiclassical approximation for the hydrogen atom (Bureeva 1968, Heim et al 1989). The expansions of the functions $J_{-s}(s \varepsilon)$ and $J_{-s}^{\prime}(s \varepsilon)$ in powers of $(1-\varepsilon) \ll 1$ are

$$
\begin{aligned}
& \mathbf{J}_{-s}(s \varepsilon)=\mathrm{J}_{-s}(s)-s(1-\varepsilon) \mathrm{J}_{-s}^{\prime}(s)+\mathrm{O}\left((1-\varepsilon)^{2}\right) \\
& \mathbf{J}_{-s}^{\prime}(s \varepsilon)=\mathrm{J}_{-s}^{\prime}(s)+(1-\varepsilon)\left[\frac{2 \sin s \pi}{\pi}+\mathrm{J}_{-s}^{\prime}(s)\right]+\mathrm{O}\left((1-\varepsilon)^{2}\right) .
\end{aligned}
$$

The functions $\mathrm{J}_{-s}(s)$ and $\mathrm{J}_{-s}^{\prime}(s)$ are strongly $s$ dependent. For small $s$ they may be evaluated by equations (17) while in asymptotic form

$$
\begin{array}{ll}
\mathbf{J}_{-s}(s)=\left(\frac{2 a}{\sqrt{3} s^{1 / 3}}\right) \cos \left(s \pi-\frac{1}{6} \pi\right) & s \gg 1 \\
\mathrm{~J}_{-s}^{\prime}(s)=\left(\frac{2 b}{\sqrt{3} s^{2 / 3}}\right) \cos \left(s \pi+\frac{1}{6} \pi\right) & s \gg 1 .
\end{array}
$$

Where

$$
a=\frac{2^{1 / 3}}{3^{2 / 3} \Gamma\left(\frac{2}{3}\right)}=0.4473 \quad b=\frac{2^{2 / 3}}{3^{1 / 3} \Gamma\left(\frac{1}{3}\right)}=0.41085 .
$$

Note the relationships $\mathbf{J}_{-s}(s)=\mathbf{J}_{s}(-s)$ and $\mathbf{J}_{-s}^{\prime}(s)=-\mathrm{J}_{s}^{\prime}(-s)$. For small $l \ll n$ it is convenient to expand expression (16) in terms of

$$
\alpha=\sqrt{1-\varepsilon^{2}}=\frac{\left(l+l^{\prime}+1\right)}{2 \sqrt{\nu \nu^{\prime}}} \ll 1 .
$$

As a result of such an expansion we finally have

$$
\left\langle\nu l|r| \nu^{\prime} l \pm 1\right\rangle=(-1)^{\Delta n} \frac{\nu \nu^{\prime}}{s}\left\{\mathrm{~J}_{-s}^{\prime}(s) \pm \alpha\left[\mathrm{J}_{-s}(s)-\frac{\sin s \pi}{s \pi}\right]+\frac{\alpha^{2}}{2}\left[\mathrm{~J}_{-s}^{\prime}(s)+\frac{3 \sin s \pi}{\pi}\right]\right\} \text {. }
$$

The functions $F_{0}(s)=-\mathrm{J}_{-s}^{\prime}(s), F_{1}=\mathrm{J}_{-s}(s)-(1 / s \pi) \sin s \pi \quad$ and $\quad F_{2}=-\left[\mathrm{J}_{-s}^{\prime}(s)+\right.$ $(3 / \pi) \sin s \pi]$ for $0<s \leqslant 2$ have been tabulated by Davydkin and Zon (1981).

\subsection{Perturbed hydrogenic atom}

Let us consider now the quasiclassical dipole matrix elements between states of the perturbed hydrogenic atom, e.g. the hydrogenic atom in a microwave field. In such a case the perturbing potential is long-range, e.g. for the hydrogenic atom in an electric field parallel to the $x$-axis, $\Delta U(r)=x F$. So, it follows from equations (10) and (14) that $\delta_{l}(r) \simeq 0$ and $\Delta(r) \simeq 0$ if $r \ll r_{2}$. In general, the dependence of $\Delta(\xi)$ on the argument in equation (15) is essential but unknown. If such a dependence in the integration 
interval of the main contribution to the integral (15) is weak, then equation (15) reduces to

$$
\begin{aligned}
\left\langle\nu l|r| \nu^{\prime} l \pm 1\right\rangle \approx & \frac{\nu \nu^{\prime}}{s}\left\{\mathrm{~J}_{s}^{\prime}(s \varepsilon) \cos \bar{\Delta}-\mathrm{E}_{s}^{\prime}(s \varepsilon) \sin \bar{\Delta}-\frac{1+\varepsilon}{\pi} \sin (\bar{\Delta}+s \pi)\right. \\
& -\frac{1-\varepsilon}{\pi} \sin \bar{\Delta} \pm \sqrt{\varepsilon^{-2}-1}\left[\mathrm{~J}_{s}(s \varepsilon) \cos \bar{\Delta}-\mathrm{E}_{s}(s \varepsilon) \sin \bar{\Delta}\right. \\
& \left.\left.+\frac{\sin \bar{\Delta}=\sin (\bar{\Delta}+s \pi)}{s \pi}\right]\right\}
\end{aligned}
$$

where $\mathrm{E}_{s}(z)$ and $\mathrm{E}_{s}^{\prime}(z)$ are the Weber function and its derivative with respect to the argument $z$.

$$
\mathrm{E}_{s}(z)=\pi^{-1} \int_{0}^{\pi} \sin (s \theta-z \sin \theta) \mathrm{d} \theta .
$$

The symbol $\bar{\Delta}$ denotes some average value of $\Delta(\xi)$ from the interval of the main contribution to the integral (15). One should notice that equation $(22 a)$ is the generalization of equation (16) for non-hydrogenic atoms. Particularly, for non-hydrogenic atoms $\bar{\Delta}=\Delta=\pi(\Delta n-s)$ and the substitution of this condition into equation (22a) yields equation (16). For the perturbed hydrogenic atom $\Delta(\xi) \rightarrow 0$ if $\xi \rightarrow 0$ and $\Delta(\xi) \rightarrow$ $\pi(\Delta n-s)$ if $\xi \rightarrow 2 \pi$. Now we will consider the cases of low and high relative transition frequency $s$. For small $s$ the main contribution to the integral (15) results from the region $\xi \sim 2 \pi$, that is why in equation $(22 a) \bar{\Delta} \approx \pi(\Delta n-s)$ and $(22 a)$ reduces to

$$
\begin{array}{rlrl}
\left\langle\nu l|r| \nu^{\prime} l \pm 1\right\rangle \simeq & (-1)^{\Delta n} \frac{\nu \nu^{\prime}}{s}\left\{J_{-s}^{\prime}(s \varepsilon)+\frac{1-\varepsilon}{\pi} \sin (s \pi)\right. & \\
& \left. \pm \sqrt{\varepsilon^{-2}-1}\left[J_{-s}(s \varepsilon)-\frac{\sin s \pi}{s \pi}\right]\right\} \quad s \leqslant 1 .
\end{array}
$$

For $s \gg 1$ the cosine and sine functions in equation (15) are rapidly oscillating if $\xi \geqslant \pi$ and the main contribution results from $\xi \ll 1$ where $\Delta(\xi) \approx 0$. Therefore in equation (22a) $\bar{\Delta} \simeq 0$ and one obtains

$\left\langle\nu l|r| \nu^{\prime} l \pm 1\right\rangle=\frac{\nu \nu^{\prime}}{s}\left\{\mathrm{~J}_{s}^{\prime}(s \varepsilon)-\frac{1+\varepsilon}{\pi} \sin s \pi \pm \sqrt{\varepsilon^{-2}-1}\left[\mathrm{~J}_{s}(s \varepsilon)-\frac{\sin s \pi}{s \pi}\right]\right\} \quad s \gg 1$.

The expansions of the functions $J_{s}(s \varepsilon)$ and $J_{s}^{\prime}(s \varepsilon)$ in powers of $(1-\varepsilon) \ll 1$ are

$$
\begin{aligned}
& \mathbf{J}_{s}(s \varepsilon)=\mathbf{J}_{s}(s)-s(1-\varepsilon) \mathrm{J}_{s}^{\prime}(s)+\mathrm{O}\left((1-\varepsilon)^{2}\right) \\
& \mathbf{J}_{s}^{\prime}(s \varepsilon)=\mathbf{J}_{s}^{\prime}(s)+(1-\varepsilon) \mathbf{J}_{s}^{\prime}(s)+\mathrm{O}\left((1-\varepsilon)^{2}\right) .
\end{aligned}
$$

While for the asymptotic forms of the functions $J_{s}(s)$ and $J_{s}^{\prime}(s)$ one can obtain the expressions (see Watson 1958, Abramowitz and Stegun 1972)

$$
\begin{aligned}
& \mathbf{J}_{s}(s)=\frac{a}{s^{1 / 3}}+\frac{\sin s \pi}{2 \pi s}-\frac{b}{70 s^{5 / 3}} \\
& \mathbf{J}_{s}^{\prime}(s)=\frac{b}{s^{2 / 3}}+\frac{a}{5 s^{4 / 3}}-\frac{\sin s \pi}{4 \pi s^{2}} \quad s \gg 1
\end{aligned}
$$

where constants $a$ and $b$ are defined by $(20 b)$.

Note that the matrix elements for the transitions between the states of the perturbed hydrogenic atom when $s \leqslant 1$, equation (23), coincide with the matrix elements for the 
non-hydrogenic atoms (16), which for the high transition frequencies, $s \gg 1$, equation (24), differ from equation (16). Such a discrepancy is caused by the range difference of the perturbing potentials $\Delta U(r)$. In the non-hydrogenic atoms the perturbing potential is of atomic origin and short range; while in the case of the perturbed hydrogenic atom, the perturbing potential $\Delta U(r)$ is external and long range. Multiphoton transitions between perturbed states of the non-hydrogenic (potassium) atom have been examined by Stoneman et al (1988).

\section{Quasiclassical dipole matrix elements for continuum states}

Dipole matrix elements for continuum-continuum transitions are required for the theoretical treatment of the above-threshold ionization phenomenon and bremsstrahlung in the ionic field. The calculations of such matrix elements are similar to those for bound-bound transitions. The wavefunction of the continuum spectrum may be written, by analogy with equation (9) (see also Delone et al 1989), as

$$
P_{k l}=\left(\frac{2}{\pi k_{\mathrm{c}}(r)}\right)^{1 / 2} \cos \left(\int_{r_{\mathrm{i}}^{\mathrm{c}}}^{r} k_{\mathrm{c}}(r) \mathrm{d} r+\delta_{l}(r)-\frac{1}{4} \pi\right) .
$$

Here $k_{c}(r)$ is the local wavenumber for the Coulomb potential

$$
\begin{aligned}
& k_{\mathrm{c}}(r)=\left(k^{2}+\frac{2}{r}-\frac{\left(l+\frac{1}{2}\right)^{2}}{r^{2}}\right)^{1 / 2}=\frac{\varepsilon k \sinh \xi}{\varepsilon \cosh \xi-1} \\
& \varepsilon=\left[1+k^{2}\left(l+\frac{1}{2}\right)^{2}\right]^{1 / 2}
\end{aligned}
$$

$k$ is the momenta related to the energy of the state in the usual way, $E=\frac{1}{2} k^{2}$, and $\varepsilon>1$ is the eccentricity of the hyperbola; while the parametric equations of motion in the continuum can be expressed as

$$
r=k^{-2}(\varepsilon \cosh \xi-1) \quad t=k^{-3}(\varepsilon \sinh \xi-\xi) \quad r_{1}^{c}=(\varepsilon-1) / k^{2} \text {. }
$$

Further calculations are analogous to those performed in section 3 . Thus, we finally find the expression for the radial dipole matrix element of transition $k l \rightarrow k^{\prime} l \pm 1$, i.e. the equivalent of equation (15)

$$
\begin{aligned}
\left\langle k l|r| k^{\prime} l \pm 1\right\rangle= & \frac{1}{\pi \bar{k}^{5}} \int_{0}^{\infty}\{\cos [s(\varepsilon \sinh \xi-\xi)+\Delta(\xi)](\varepsilon-\cosh \xi) \\
& \left. \pm \sqrt{\varepsilon^{2}-1} \sin [s(\varepsilon \sinh \xi-\xi)+\Delta(\xi)] \sinh \xi\right\}(\varepsilon \cosh \xi-1) \mathrm{d} \xi
\end{aligned}
$$

where

$s=\frac{\Delta E}{\bar{k}^{3}} \quad \varepsilon=\left[1+k k^{\prime}\left(\bar{l}+\frac{1}{2}\right)^{2}\right]^{1 / 2} \quad \bar{k}=\left(k k^{\prime}\right)^{1 / 2} \quad \tilde{l}=\frac{1}{2}\left(l+l^{\prime}\right)$.

The analysis of expression ( $30 a$ ) is similar to the analysis of equation (15).

For the non-hydrogenic atom $(\Delta(\xi)=\Delta)$ after integration by parts one obtains the limiting case $|s| \ll 1$

$$
\left\langle k l|r| k^{\prime} l \pm 1\right\rangle=\frac{k}{\pi \omega^{2} \varepsilon}\left[\cos \Delta \pm\left(\bar{l}+\frac{1}{2}\right) k\left(s \cos \Delta \ln \frac{2 k^{3}}{\gamma \omega \varepsilon}-\sin \Delta\right)\right]
$$

where $\omega=|\Delta E|, \gamma=\mathrm{e}^{C}=1.781 \ldots$, and $C$ is the Euler constant. 
The expansion of equation $(30 a)$ in terms of $\alpha=\left(\varepsilon^{2}-1\right)^{1 / 2}$ in the asymptotic limit $|s| \gg 1$ is

$$
\left\langle k l|r| k^{\prime} l \pm 1\right\rangle=\frac{2}{\sqrt{3} \omega^{5 / 3}}\left[b \cos \left(\frac{s}{|s|} \Delta-\frac{\pi}{6}\right) \pm \alpha \frac{a s}{|s|^{2 / 3}} \cos \left(\frac{s}{|s|} \Delta+\frac{\pi}{6}\right)\right] .
$$

For the hydrogen atom $(\Delta=0)$ performing a partial integration of equation $(30 a)$ twice yields the result following from the Heisenberg correspondence principle which relates the matrix element with the Fourier component of the hyperbolic motion (Landau and Lifshitz 1977)

$$
\left\langle k l|r| k^{\prime} l \pm 1\right\rangle=\frac{1}{2 \bar{k}^{5} s}\left(H_{\mathrm{i} s}^{(1) \prime}(\mathrm{i} s \varepsilon) \pm \mathrm{i} \frac{\left(\varepsilon^{2}-1\right)^{1 / 2}}{\varepsilon} H_{\mathrm{is}}^{(1)}(\mathrm{i} s \varepsilon)\right)
$$

where $H_{\nu}^{(1)}(z)$ is the Hankel function of the first kind. The limiting cases of equation (33) are the expressions (31) and (32) with $\Delta=0$. It should be noted that equations (30)-(32) are the generalization for the non-hydrogenic atoms and $\alpha \neq 0$ of the corresponding expressions by Delone et al (1989)†.

\section{The one-dimensional atom}

In the past few years much theoretical and experimental work has been devoted to the investigation of highly excited atoms in microwave fields. The simplest approximation of this system is the one-dimensional hydrogen atom model, which conveniently describes an actual hydrogenic atom prepared in a very excited state (see Casati et al 1987,1988 ) as well as the surface-state electrons on liquid helium (Jensen 1984). The Hamiltonian of the one-dimensional hydrogen atom is

$$
H_{0}= \begin{cases}\frac{1}{2} p^{2}-(1 / x) & x>0 \\ \infty & x \leqslant 0\end{cases}
$$

while the wavefunctions $\Psi_{n}(x)$ can be expressed as

$$
\Psi_{n}(x)=x R_{n 0}(x)=P_{n 0}(x)
$$

i.e. they coincide with the solutions $P_{n 0}(x)$ of the radial Schrödinger equation of the hydrogen atom for $s$-states (Jensen 1984). The parametric equation of motion for $x$ is

$$
x=\nu^{2}(1-\cos \xi) \quad t=\nu^{3}(\xi-\sin \xi) .
$$

That is why the expressions for the matrix elements of the one-dimensional atoms follow from the results given above. Requiring only to use in all equations above $\varepsilon=1$ and to change the sign of matrix element; because for the one-dimensional atom $r=x$ equals $-x$ of the three-dimensional atom with $\varepsilon=1$ (compare (36) with (39)). Therefore, the dipole matrix elements for the one-dimensional atom are

$$
\left\langle\nu|x| \nu^{\prime}\right\rangle=-\left.\left\langle\nu l|r| \nu^{\prime} l^{\prime}\right\rangle\right|_{k=1, l=l^{\prime}=0} .
$$

One should notice that as the really one-dimensional atom, $\Delta=\delta^{\prime}-\delta$ is usually a small quantity as the perturbation of the same symmetry states, especially with near energies, does not vary much.

$\dagger$ Note the wrong factor in equation (4) and the second term in equation (7) by Delone et al (1989). Analysis of the semiclassical matrix elements for the dipole transitions in the atomic hydrogen has been made by Trippenbach et al (1989). 


\section{Classical dynamics of the one-dimensional hydrogen atom in a harmonic field}

Dipole matrix elements between the states of high principal quantum numbers are closely related to the energy change of a classical atom in a harmonic field and with the stochastic diffusive ionization of the hydrogenic atom. However, as was shown by Gontis and Kaulakys (1987), the energy change of the electron in a harmonic field during the intrinsic motion period depends on the initial coordinate of the electron, i.e. the energy change between two subsequent passages at the aphelion $r_{2}$ differs from the energy change between two subsequent passages at the perihelion $r_{1}$. Note that the energy change is proportional to the Fourier component of the electron velocity (Gontis and Kaulakys 1987). On the other hand, quantal transition amplitudes between the photonic states may be expressed in terms of Fourier components of the electron coordinate (Percival and Richards 1970, Gontis and Kaulakys 1991). But, according to the Heisenberg correspondence principle, Fourier components are related to the

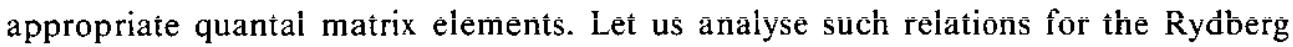
atom.

The radial dipole matrix elements (13) and (15) may be expressed in the form (see also Davydkin and Zon 1981)

$$
\begin{aligned}
\left\langle\nu l|r| \nu^{\prime} l \pm 1\right\rangle & =\frac{2}{T} \int_{0}^{T / 2} r(t) \cos \left[\omega_{\nu^{\prime} \nu} t \mp \varphi(t)+\Delta\right] \mathrm{d} t \\
& =\frac{2}{T}\left(\int_{0}^{T / 2} x(t) \cos \left[\omega_{\nu^{\prime} \nu} t+\Delta\right] \mathrm{d} t \pm \int_{0}^{T / 2} y(t) \sin \left[\omega_{\nu^{\prime} \nu} t+\Delta\right] \mathrm{d} t\right) .
\end{aligned}
$$

Here $T=2 \pi \nu^{3}$ is the period of the electron intrinsic motion, $\omega_{n \nu^{\prime}}=E_{y^{\prime}}-E_{\nu}$, and $\varphi$ is the polar angle while $x$ and $y$ are the Cartesian coordinates of the electron. The parametric equations of motion for $x, y$ and $\varphi$ are

$$
\begin{array}{ll}
x=\nu^{2}(\cos \xi-\varepsilon) & y=\nu^{2}\left(1-\varepsilon^{2}\right)^{1 / 2} \sin \xi \\
\varphi=\cos ^{-1}\left(\frac{\cos \xi-\varepsilon}{1-\varepsilon \cos \xi}\right) & t=\nu^{3}(\xi-\sin \xi) .
\end{array}
$$

For the one-dimensional hydrogenic, perturbed hydrogenic or non-hydrogenic atoms according to expression (38) we have

$$
\begin{aligned}
\left\langle\nu|x| \nu^{\prime}\right\rangle & =\frac{2}{T} \int_{0}^{T / 2} x(t) \cos \left[\omega_{\nu^{\prime} \nu^{\prime}} t+\Delta\right] \mathrm{d} t \\
& =\frac{2}{\nu \pi} \sin (s \pi+\Delta)-\frac{1}{\pi s} \int_{0}^{T / 2} \dot{x}(t) \sin \left[\omega_{\nu^{\prime} \nu^{\prime}} t+\Delta\right] \mathrm{d} t
\end{aligned}
$$

where the parametric equation of motion for $x$ is given by expressions (36). For the hydrogenic atom in equation $(40), \Delta=\pi(\Delta n-s)$. According to section 3.2 the same is true for the perturbed hydrogenic atom if $s \leqslant 1$. We see from equations (38) and (40) that the matrix elements are equal to the Fourier sine or cosine (with appropriate phases) components taken for half of the period.

As was noticed above the perturbation of the classical atom in a harmonic field is connected to the Fourier components as well, i.e. with expressions similar to equation (40). Let us analyse such perturbation in detail. 
In the dipole approximation the interaction of the atom with the electromagnetic field is

$$
V=x F_{0} \cos (\omega t+\vartheta)
$$

where $F_{0}, \omega$ and $\vartheta$ are the electric field amplitude strength, angular frequency and phase, respectively. The quantal transition amplitudes between the strongly coupled highly excited states may be expressed in terms of the classical action function of the perturbing potential taken over an unperturbed path (Percival and Richards 1970, Gontis and Kaulakys 1991). For half of the intrinsic motion period the classical action functions of the perturbing potential (41) are

$$
\begin{aligned}
\Delta U\left(-\frac{1}{2} T, 0\right) \equiv & F_{0} \int_{-T / 2}^{0} x(t) \cos (\omega t+\vartheta) \mathrm{d} t \\
= & -\frac{2 \nu^{2} F_{0}}{\omega} \sin (-\pi s+\vartheta)-\frac{\pi \nu^{2} F_{0}}{\omega}\left[\mathrm{J}_{s}^{\prime}(s) \cos \vartheta+\mathrm{E}_{s}^{\prime}(s) \sin \vartheta\right] \\
\Delta U\left(0, \frac{1}{2} T\right)= & \frac{2 \nu^{2} F_{0}}{\omega} \sin (\pi s+\vartheta)-\frac{\pi \nu^{2} F_{0}}{\omega}\left[\mathrm{J}_{\mathrm{s}}^{\prime}(s) \cos \vartheta-\mathrm{E}_{s}^{\prime}(s) \sin \vartheta\right] \\
\Delta U\left(\frac{1}{2} T, T\right)= & -\frac{2 \nu^{2} F_{0}}{\omega} \sin (\pi s+\vartheta) \\
& -\frac{\pi \nu^{2} F_{0}}{\omega}\left[\mathrm{J}_{s}^{\prime}(s) \cos (\vartheta+2 \pi s)+\mathrm{E}_{s}^{\prime}(s) \sin (\vartheta+2 \pi s)\right]
\end{aligned}
$$

while for the complete period we have

$$
\begin{aligned}
& \Delta U\left(-\frac{1}{2} T, \frac{1}{2} T\right)=\frac{4 \nu^{2} F_{0}}{\omega} \sin \pi s \cos \vartheta-\frac{2 \pi \pi \nu^{2} F_{0}}{\omega} \mathbf{J}_{s}^{\prime}(s) \cos \vartheta \\
& \Delta U(0, T)=-\frac{2 \pi \nu^{2} F_{0}}{\omega} \mathbf{J}_{-s}^{\prime}(s) \cos (\pi s+\vartheta) .
\end{aligned}
$$

Thus, the classical action function taken over an unperturbed path for the period of intrinsic motion depends on the initial coordinate of the electron.

For the derivation of mapping (iterative) forms of the classical equations of motion for hydrogenic atom in an oscillating electric field it is necessary to evaluate the energy change $\Delta E$ of the electron during the period of intrinsic motion (Gontis and Kaulakys 1987). $\Delta E$ may be evaluated according to the classical perturbation theory (Lichtenberg and Lieberman 1983) from the equation of motion

$$
\dot{E}=\mathscr{F} \dot{x}
$$

where $\mathscr{F}=-\partial V / \partial x$ is the force acting on the electron. Substitution of the potential (41) into equation (47) gives

$$
\dot{E}=-\dot{x} F_{0} \cos (\omega t+\vartheta)
$$

Integration of equation (48) for half of the intrinsic motion period yields

$$
\begin{aligned}
\Delta E\left(-\frac{1}{2} T, 0\right) & =-F_{0} \int_{-T / 2}^{0} \dot{x}(t) \cos (\omega t+\vartheta) \mathrm{d} t \\
& =\pi \nu^{2} F_{0}\left[\mathrm{~J}_{s}^{\prime}(s) \sin \vartheta-\mathrm{E}_{s}^{\prime}(s) \cos \vartheta\right] \\
\Delta E\left(0, \frac{1}{2} T\right)= & \pi \nu^{2} F_{0}\left[\mathrm{~J}_{s}^{\prime}(s) \sin \vartheta+\mathrm{E}_{s}^{\prime}(s) \cos \vartheta\right] \\
\Delta E\left(\frac{1}{2} T, T\right) & =\pi \nu^{2} F_{0}\left[\mathrm{~J}_{s}^{\prime}(s) \sin (2 \pi s+\vartheta)-\mathrm{E}_{s}^{\prime}(s) \cos (2 \pi s+\vartheta)\right]
\end{aligned}
$$


while for the complete period we have (Gontis and Kaulakys 1987)

$$
\begin{aligned}
& \Delta E\left(-\frac{1}{2} T, \frac{1}{2} T\right)=2 \pi \nu^{2} F_{0} \mathrm{~J}_{s}^{\prime}(s) \sin \vartheta \\
& \Delta E(0, T)=2 \pi \nu^{2} F_{0} \mathrm{~J}_{-s}^{\prime}(s) \sin (\pi s+\vartheta) .
\end{aligned}
$$

In equations (42)-(53)

$$
s=\omega \nu^{3} \quad \nu=(-2 E)^{-1 / 2}
$$

and $E$ is the energy of the electron.

One should notice the similarity of the expressions $(45)-(46)$ and $(52)-(53)$ to the semiclassical expressions for the matrix elements of the perturbed hydrogenic atom (equations (23) and (24) in accordance with equation (37)). As one can see from expressions (38) and (40), the matrix elements are related to the appropriate Fourier components of the electron coordinate and velocity taken for half of the intrinsic motion period. The comparison of expressions (45)-(46) and (52)-(53) with expressions (23) and (24) according with equation (40) shows that the matrix elements of the perturbed hydrogenic atom may also be expressed in terms of the appropriate Fourier components for the complete period of intrinsic motion.

We see from equations (52) and (53) that the energy change of the electron during the period of intrinsic motion depends on the initial coordinate of the electron (Gontis and Kaulakys 1987) and for motion between two subsequent passages at the perihelion may be three times larger than for motion between two subsequent passages at the aphelion. However, equations (49)-(53) are only the perturbative approximation because, in general, the energy change depends on the initial energy of the electron (in terms of $\nu$ and $s$ ). So, equations (49)-(51) are more precise than equations (52)-(53). That is why the integration interval for equations (49)-(51) is twice as short than for equations (52)-(53). But in the high field frequency limit

$$
\mathrm{J}_{s}^{\prime}(s)=b / s^{2 / 3} \quad \mathrm{E}_{s}^{\prime}(s)=-b / 3^{1 / 2} s^{2 / 3} \quad b \simeq 0.41085 .
$$

So, the energy changes $\Delta E\left(-\frac{1}{2} T, 0\right)$ and $\Delta E\left(0, \frac{1}{2} T\right)$, according to equations (49) and (50), are energy independent and equations (49) and (50) may be added up. Therefore, for high frequencies, expression (52) is as exact as equations (49) and (50). On the contrary, expression (53) is less precise than equations (50) and (51) for all values of frequency (due to the energy dependency of the energy change $\Delta E\left(\frac{1}{2} T, T\right)$ according to equation (51)).

Therefore, for low and medium frequencies of the harmonic field, equations (49)-(51) for the electron's energy change during half of the intrinsic motion period represent more precisely the influence of the harmonic field on the excited atom than equations (52) and (53) for the complete period. This is especially significant for the strong electric fields. On the other hand, the stochastization process of the electron motion in a low frequency field takes place mainly in the relatively strong field, i.e. near the validity limit of the classical perturbation theory (see, e.g. Gontis and Kaulakys 1987 and references therein). Moreover, at low frequencies the classical theory describes well the stochastic dynamics of the hydrogenic atom in a microwave field (Casati et al 1987, 1988). So, the specification of the mapping equations of motion for the hydrogenic atom in a harmonic field, given in papers by Gontis and Kaulakys (1986, 1987 ) is desirable. More precise iterative equations of motion may be obtained by analogy with Gontis and Kaulakys (1987) after scaling of the energy and electric field 
amplitude in equations (49)-(51). As a result of this procedure one can write

$$
\begin{aligned}
& \varepsilon_{k+1}=\varepsilon_{k}-2 \pi \Phi_{0} \varepsilon_{0}^{2} \varepsilon_{k}^{-1}\left(J_{s_{k}}^{\prime}\left(s_{k}\right) \sin \vartheta_{k}-\mathrm{E}_{s_{k}}^{\prime}\left(s_{k}\right) \cos \vartheta_{k}\right) \\
& \varepsilon_{k+2}=\varepsilon_{k+1}-2 \pi \Phi_{0} \varepsilon_{0}^{2} \varepsilon_{k+1}^{-1}\left(J_{s_{k+1}}^{\prime}\left(s_{k+1}\right) \sin \vartheta_{k}+\mathrm{E}_{s_{k+1}}^{\prime}\left(s_{k+1}\right) \cos \vartheta_{k}\right) \\
& \vartheta_{k+2}=\vartheta_{k}+2 \pi / \varepsilon_{k+2}^{3 / 2} .
\end{aligned}
$$

Here $\varepsilon=s^{-2 / 3}=-2 E / \omega^{2 / 3}$ and $\Phi_{0}=F_{0} / 4 E_{0}^{2}$ are the scaled energy and electric field amplitude, respectively, with $E_{0}$ being the initial energy of the electron.

The approximation of equations (56) for high frequencies or weak fields is equivalent to equations (10) by Gontis and Kaulakys (1987). These equations have been the first mapping form of the classical equations of motion for the hydrogenic atom in a harmonic field (see also Gontis and Kaulakys 1986) which greatly facilitated the numerical investigation of the stochasticity and the ionization process. On the basis of mapping equations the analytical estimations of the threshold field strengths for the onset of chaos, the diffusion coefficient for the electron in the energy space and the mean time for the classical diffusion ionization have been fulfilled. In addition, for the first time has been predicted the resonance structure in the dependence of the threshold field strengths on the frequency of the field in the high frequency region. Recent experiments (Galvez et al 1988) revealed such a resonance structure, though the quantum analysis of the ionization process in this case is more convenient.

The system of equations (56) contains, as some approximations, both cases considered in the paper by Gontis and Kaulakys (1987), i.e. the electron's energy changes between two subsequent passages of the aphelion as well as between two subsequent passages of the perihelion (equations (6) and (22) of Gontis and Kaulakys 1987, respectively), but in fact it is more precise than those partial cases. This is the case, since one can as an approximation add up in equation (56) any of two subsequent energy changes for the half of the period, e.g. either $\varepsilon_{k+1}-\varepsilon_{k}$ and $\varepsilon_{k+2}-\varepsilon_{k+1}$ or $\varepsilon_{k+2}-\varepsilon_{k+1}$ and $\varepsilon_{k+3}-\varepsilon_{k+2}$. As equations (56) represent the interaction process of the atom with a microwave field more exactly and in more detail they may render more precisely and entirely the resonance structure of the atom-field interaction and the stochasticity feature for low and medium relative frequencies of the field $(s \leqslant 1)$. System (56) is rather complex for an analytical study but it may be transformed to the area-preserving case (see for analogy Gontis and Kaulakys 1987) and investigated numerically. However, this is beyond the scope of this paper. In this section the relationship between the dipole matrix elements and the energy change of the classical atom in a harmonic field has been revealed and analysed and in addition the improved mapping equations of motion for the classical hydrogenic atom in a harmonic field have been derived.

\section{Conclusions}

Fulfilled theoretical analysis of the analytical expressions for dipole matrix elements between high atomic states with fractional effective principal quantum numbers shows the dependence of the matrix elements and transition probabilities on the nature of perturbation for the Coulomb potential. Thus, for the non-hydrogenic atoms the deviation of the atomic core potential from the Coulomb potential is appreciable only at small distances while for the hydrogenic atom in an electromagnetic field the perturbing potential is long-range. This difference results in different expressions for 
the matrix elements of the non-hydrogenic atoms and perturbed hydrogenic atom. One can conclude from this, that analytical wavefunctions in the effective potential model (see, e.g. Kostelecky and Nieto 1985) are not general results for the non-hydrogenic atoms but only one possible approximation.

The theoretical investigation is performed for discrete and continuum states as well as for the one-dimensional atom. The obtained results generalize and increase the accuracy of some known expressions for the hydrogenic and non-hydrogenic atoms and extend them for the perturbed hydrogenic atom.

Furthermore, the analysis of the classical dynamics of the one-dimensional hydrogen atom in a harmonic field is fulfilled. The connection between the energy change of the classical atom in a harmonic field and expressions for the dipole matrix elements is revealed and the accurate iterative mapping equations of motion for the classical hydrogen atom in an oscillating electric field are derived. One can conclude that the mapping form of the classical equations of motion of the hydrogenic atom in a harmonic field, derived for the first time by Gontis and Kaulakys $(1986,1987)$ and widely used (see, e.g. Casati et al 1987, 1988, Koch et al 1989 and references therein) is exact in the high field frequency limit only, while for low and medium frequencies more precise equations (56) must be used.

The results of the paper may contribute to the deeper understanding of the quantumclassical correspondence principle and adequate quasiclassical theory for Rydberg atoms as well as to the development of the stochastic dynamics theory of the hydrogenic atom in a microwave field.

\section{Acknowledgment}

The author is grateful to the A v Humboldt Foundation for a research fellowship.

\section{References}

Abramowitz M and Stegun I A 1972 Handbook of Mathematical Functions ed I A Stegun (New York: Dover) Beigman I L and Bureeva L A 1981 Izv. AN SSSR Ser. Fiz. 45 2277-88

Bethe H A and Salpeter E E 1957 Quantum mechanics of one and two electron atoms Handbuch der Physik vol 35 (Berlin: Springer)

Bureeva L A 1968 J. Sov. Phys.-Astron. 45 1215-21

Casati G, Chirikov B V, Shepelyansky D L and Guarneri 11987 Phys. Rep. 154 77-123

Casati G, Guarneri I and Shepelyansky D L 1988 IEEE J. Quantum. Electron. QE-24 1420-44

Davydkin V A and Zon B A 1981 Sov. Phys.-Opt. Spectrosc. 51 13-5

Delone N B, Goreslavsky S P and Krainov V P 1989 J. Phys. B: At. Mol. Opt. Phys. 22 2941-5

Galvez E J, Sauer B E, Moorman L, Koch P M and Richards D 1988 Phys. Rev. Lett. 61 2011-4

Gontis V and Kaulakys B 1986 Sov. Phys.-Collect, $27111-3$ (deposited in VINITI as no 5087-V86)

— 1987 J. Phys. B: At. Mol. Phys. 20 5051-64

1991 Lies. Fiz. Rinkinys. 31 127-32

Gordon W 1929 Ann. Phys. Lpz. 2 1031-56

Heim T A, Trautmann D and Baur G 1989 J. Phys. B: At. Mol. Opt. Phys. 22 727-40

Jensen R V 1984 Phys. Rev. A 30 386-97

Koch P M, Moorman L, Sauer B E, Galvez E J, van Leeuwen K A H and Richards D 1989 Phys. Scr. T 26 51-8

Kostelecky V A and Nieto M M 1985 Phys. Rev. A 32 3243-6

Landau L D and Lifshitz E M 1960 Quantum Mechanics (Oxford: Pergamon)

1977 Field Theory vol 2 (London: Pergamon) 
Lichtenberg A J and Lieberman M A 1983 Regular and Stochastic Motion (New York: Springer)

Percival I C and Richards D 1970 J. Phys. B: At. Mol. Phys. 3 1035-46

Stoneman R C, Thomson D S and Gallagher T F 1988 Phys. Rev. A $371527-40$

Trippenbach M, Rzazewski K, Feodorov M V and Kazakov A E 1989 J. Phys. B: At. Mol. Opt. Phys. 22 1193-1205

Watson G N 1958 A Trietise on the Theory of Bessel Functions (Cambridge: Cambridge University Press) 